\title{
Chiari Malformation Type 1 in Adults Managed by Surgical Decompression: New Prospective
}

\author{
Amr Abdelmonam Mostafa Elkatatny*, Mohamed H. Aly \\ Department of Neurosurgery, Faculty of Medicine, Cairo University, Giza, Egypt \\ Email: ^amramostafa2013@gmail.com
}

How to cite this paper: Elkatatny, A.A.M. and Aly, M.H. (2020) Chiari Malformation Type 1 in Adults Managed by Surgical Decompression: New Prospective. Open Journal of Modern Neurosurgery, 10, 382-391. https://doi.org/10.4236/ojmn.2020.103041

Received: May 9, 2020

Accepted: July 14, 2020

Published: July 17, 2020

Copyright (อ 2020 by author(s) and Scientific Research Publishing Inc. This work is licensed under the Creative Commons Attribution International License (CC BY 4.0).

http://creativecommons.org/licenses/by/4.0/ (c) (i) Open Access

\begin{abstract}
Aim of the work: This study aims to assess the value of the surgical management in the improvement of the symptoms \& signs of patients with Chiari malformation type 1 and radiological follow up in adults. Patients and methods: This study included 30 consecutive patients with Chiari malformation type I who were indicated for surgery at neurosurgery department. Data were collected prospectively from the involved patients who were evaluated preoperatively and underwent evaluation by CT scanning of the brain \& skull and MRI imaging of the brain and spine. CT and MRI were done as the routine follow up investigations for all patients. We operated through midline suboccipital craniectomy, durotomy in y shaped manner, shrinkage of cerebellar tonsils by bipolar electrocautery, duroplasty by fascia lata graft, watertight closure. Results: The assessment from E. J. N. S. (Egyptian Journal of Neurosurgery) vol. 24 no. 2 June 2009 used for evaluating the patients clinically. 18 patients reported good outcome, 6 fair, 6 poor. We evaluated the size of the syrinx if present preoperative in follow up. 15 (50\%) patients showed marked reduction (more than or equal to 60\%) in size of syrinx, $3(10 \%)$ mild reduction (less than or equal to $30 \%$ ) in size and $12(40 \%)$ with no change. Conclusion: The Chiari type 1 malformation constitutes a controllable malformation with good outcomes. With current microsurgical techniques, the results of the bony decompression and duroplasty became excellent. Before undergoing surgical treatment for CM-I, symptomatic patients and their families should be given clear information about the success of treatment and potential complications.
\end{abstract}

\section{Keywords}

Chiari Malformation Type 1, Hydrocephalus, Posterior Fossa

Decompression, Duroplasty, Water Tight Closure, Fascia Lata 


\section{Introduction}

The four traditional types of Chiari malformations represent varying degrees of involvement of rhombencephalic derivatives. Three of these (type I to III) have progressively more severe herniation of these structures outside the posterior fossa as a common feature. These three also have in common a pathogenesis that involves a loss of free movement of cerebral spinal fluid (CSF) out of the normal outlet channels of the fourth ventricle. Although a large majority of hindbrain hernias are congenital, acquired CIMs occur and are not rare. Other causes that may be considered are intracranial tumor, or other mass lesions, especially within the posterior fossa [1].

\section{Patients \& Methods}

This study included 30 consecutive surgically indicated patients with Chiari malformation type I. They were operated in Neurosurgery Department, Kasr AlAini Cairo University Hospitals (March 2018-December 2019). Data were collected prospectively from the involved patients in the study. All patients included in the series were evaluated preoperatively by neurological, ophthalmological and radiological assessment.

Patients with Chiari malformation type 1 included are (18 - 65) years old, Symptomatic with progressive course, rapid neurological deterioration and no high risk medical condition. We excluded out of our age range (18 - 65) years old, 1) asymptomatic cases, 2) symptomatic with stationary course, 3) no neurological deficit and 4) high risk medical condition. For the purpose of comparison, all data regarding the patients will be reviewed including: age, sex, Past and medical history, drug intake, mode of onset, duration and course of the current illness. The patients were asked about the following symptoms particularly; headache, vomiting, visual affection (diminution of vision, blurring of vision), sphincter disturbances, heaviness and tingling and numbness. Full general, neurological and ophthalmological examinations on admission were done. All patients had evaluation by CT scanning of the brain \& skull and MRI imaging of the brain. CT and MRI were done as the routine follow up investigations for all patients .Follow up CT was done after 24 hours for all patients. Follow up MRI was 6 months postoperatively. Extra scans were done for any deteriorating patient.

Patients were informed about the underlying neurological problem, role of surgery, surgical technique, post-operative care and expected (mortality \& morbidity and their percentages). The consent should be detailed and written.

Each case included in this study was given antibiotics (third generation cephalosporin) at the induction of the anesthesia (2 gm), and continue for at least one week after surgery ( 1 gm every 12 hours).

We operate through midline suboccipital craniectomy, durotomy in y shaped manner, shrinkage of cerebellar tonsils bipolar electrocautery, and duroplasty by fascia lata graft, watertight closure. 
Monitoring of the patient continues postoperatively including the vital signs, conscious level and for detection of any complications. Analgesics are administered as needed. CT scan is usually performed on the day after the operation to check for brain edema, any hematoma, pneumocephalus, and extent of bony decompression. The immediate postoperative data was evaluated in all patients and then patients were followed subsequently as outpatient, 3 - 6 months later the evaluation was done both clinically regarding any improvement or deterioration in the symptomatology and radiologically (MRI \& CT scan) to detect any ventriculomegaly, enlargement of syrinx.

The study was approved by the Ethics Board of Faculty of Medicine, Cairo University.

\section{Results}

We reviewed 30 cases of Chiari type 1 malformation treated surgically between May 2018 and December 2019 at Neurosurgery Department of Kasr AlAiny, Cairo University Hospitals. The mean age at the time of surgery for the studied group was 41.5 years ranging (18 to 65) as we see in Figure 1. As in Figure 2, there was a female predominance. There were 18 females (60\%) and 12 males (40\%) which provide a female/male ratio of (1.5/1).

Figure 3 demonstrates the clinical presentations. The most common clinical findings presented at diagnosis were headache in 30 patients (100\%), neck pain in 27 patients (90\%), dizziness and fatigue in 21 (70\%), extremities numbness and paresthesia in 18 patients $(60 \%)$, weakness of fine movements in 12 patients (40\%), sphincter manifestation in 6 patients $(20 \%)$, cerebellar dysfunction in 6 patients (20\%).

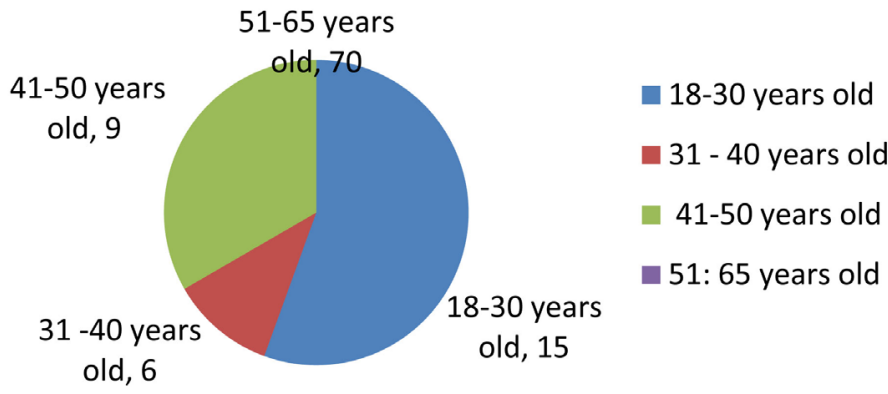

Figure 1. Pie chart showing the age of patients at diagnosis.

\section{sex distribution}

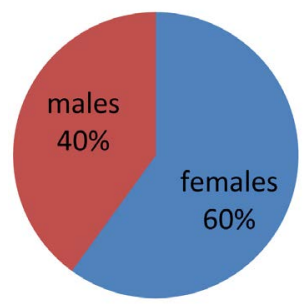

Figure 2. Pie chart showing female predominance. 


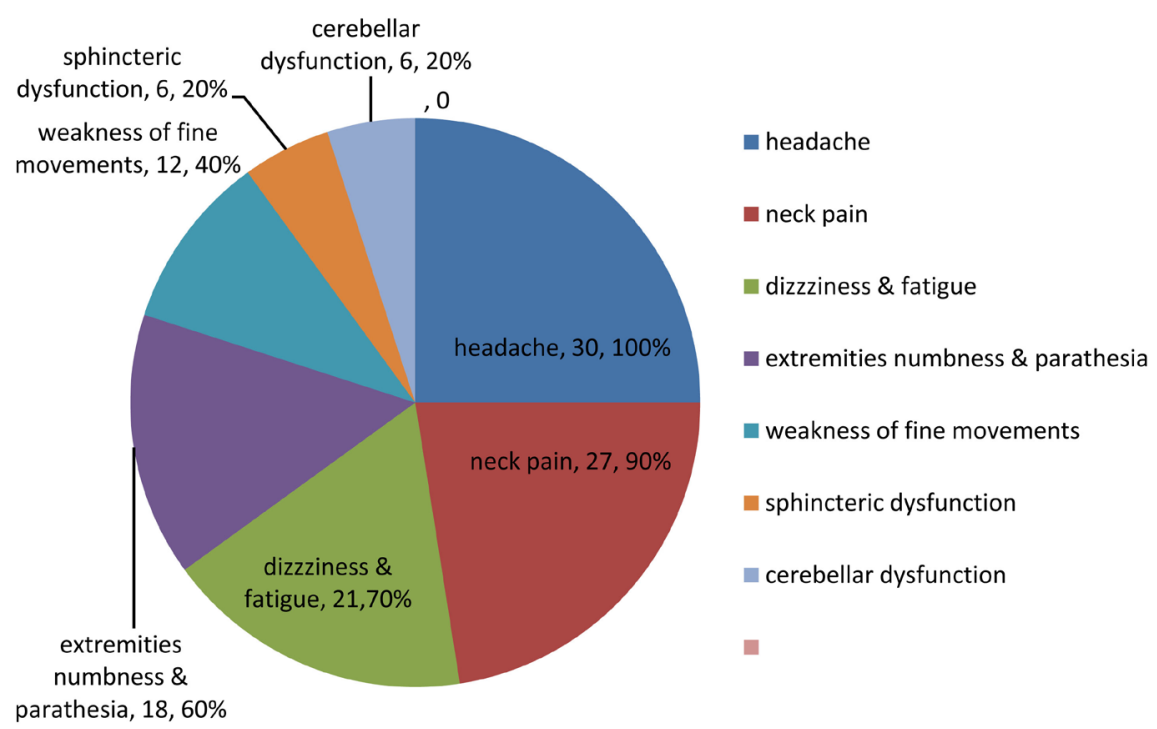

Figure 3. Pie chart showing the main presentation at diagnosis.

The Clinical outcome was assessed as: 1) Good: patients have marked improvement with no residual symptoms causing any sort of disability, 2) Fair: patients have residual symptoms causing mild disability in performing their usual daily activities, 3) Poor: patients have no improvement at all or worsening of symptoms and demonstrated in Figure 4. This assessment is from $E$. J. N. S. vol. 24 no. 2 June 2009. In our study, 18 (60\%) patients reported marked improvement after surgery, 6 (20\%) reported fair improvement \& $6(20 \%)$ were poor.

Regarding the size of the syrinx as in Figure 5, $15(50 \%)$ patients showed marked reduction (more than or equal to $60 \%$ ) in size, $3(10 \%)$ mild reduction (less than or equal to $30 \%$ ) in size and $12(40 \%)$ with no change.

We evaluated the lower border of the tonsils after shrinkage by bipolar electrocautery in follow up in Figure 6. We found $24(80 \%)$ patients showed normal level, and 6 (20\%) patients were within $5 \mathrm{~mm}$ below foramen magnum.

In this series, the median complication rate was $6.2 \%$. The complications most commonly reported were CSF leak ( 2 cases) $6.66 \%$, pseudomeningocele (4 cases) $13.3 \%$, aseptic meningitis (2 cases) $6.66 \%$, wound infection (1 case) $3.33 \%$, meningitis (1 case) $3.33 \%$, neurological deficit (2 cases) 6.66\%, hydrocephalus and respiratory dysfunction ( 1 case) $3.33 \%$, postoperative headaches (5 cases) $16.7 \%$ and no epidural/subdural hematoma of the posterior cranial fossa. Mortality was reported for 1 of 30 adult patients (3.33\%). The reported etiology was pneumonia/respiratory failure, infection/sepsis. Figure 7 demonstrates different complications.

\section{Discussion}

In this study, Chiari type 1 malformation was found to affect females more than males. In this study 18 females (60\%) were affected against 12 males (40\%), with a female/male ratio $1.5 / 1$. The age distribution ranged (18 - 30), was the major area with 15 patients (50\%). 9 patients (30\%) were in the age group, which 


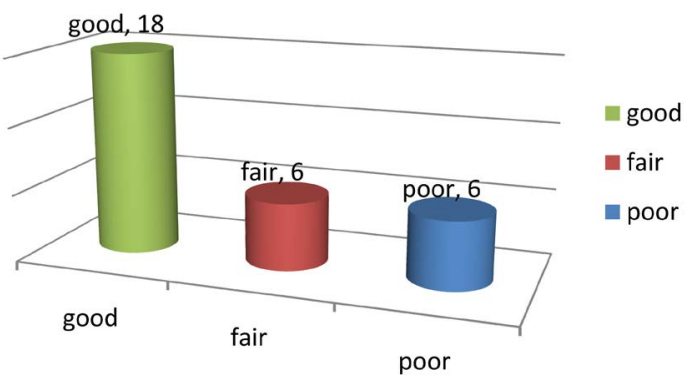

Figure 4. Showing the clinical outcome after surgery.

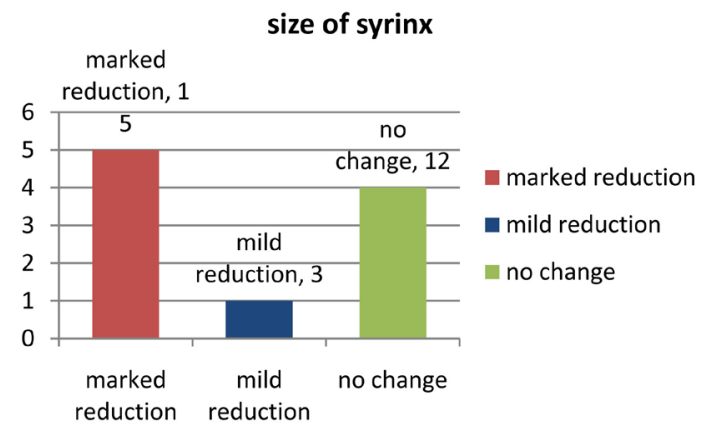

Figure 5. Showing the size of syrinx postoperative.

level of the tonsils radiologically post-operative

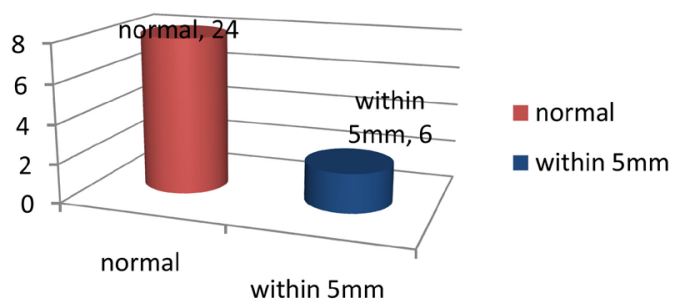

Figure 6. Showing the level of the tonsils postoperative.

\section{POSTOPERATIVE COMPLICATIONS}

POSTOPERATIVE HEADACHE HYDROCEPHALUS AND RESPIRATORY DYSFUNCTION NEUROLOGICAL DEFICIT

WOUND INFECTION

MENINGITIS

ASEPTIC MENINGITIS

PSEUDOMENINGIOCELE

CSF LEAK

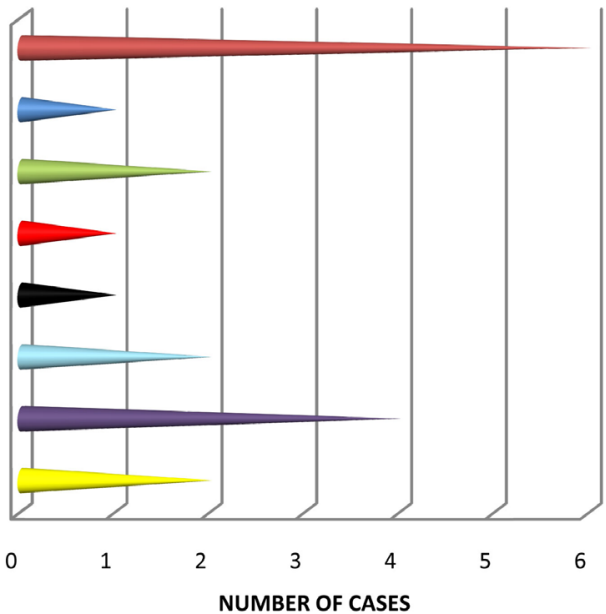

Figure 7. Showing the different complications. 
extended from 41 to 50 years. In previous series, there was also female predominance and most cases occurred in adolescence [2].

The most common clinical findings presented at diagnosis among our patients were headache in all patients, neck pain in 27 patients, dizziness and fatigue in 21 cases, extremities numbness and paresthesia in 18 cases. These are close to the study in which headache occurred in $100 \%$ of cases, neck pain in $89 \%$ of cases, dizziness and fatigue in $83 \%$, numbness in $72 \%$ [3].

In an evaluation of operative procedures for clinical outcome after decompression, suboccipital headaches aggravated by the Valsalva maneuver are one of the highlighted symptoms in all patients with CM-I [4]. However, only $16.7 \%$ of this series reported postoperative headache. As the other aspects of postoperative results showed, the improvement or resolution of headaches was also good, occurring in $73 \%$ of adult patients [5].

In our study, 18 (60\%) patients reported marked improvement after surgery, 6 (20\%) reported fair improvement \& $6(20 \%)$ were poor. In other studies, preoperative neurological deficits improved or resolved in $73 \%$ of the adult series, $84 \%$ of pediatric series, $72 \%$ of combined series, and $75 \%$ for all series [5]. These satisfactory neurological results in approximately three-quarters of all patients correspond with the similarly good improvement/resolution of syrinx [6]. However, the difference in the results of worsening neurological deficits in the follow-up period ( $7 \%$ vs $1 \%$, adult vs pediatric, respectively) that this difference is because younger patients frequently responded better to surgical decompression [5]. It is well established that CM-I is the leading cause of syringomyelia and that the creation and worsening of syringomyelia is one of the main causes of neurological symptoms and deficits in these patients. The improvement or resolution of syringomyelia is one of the main goals of the surgical treatment of patients with CM-I [7]. Improvement/resolution of syringomyelia was described in all of the reported series (pediatric, adult, combined, and total) for almost three-quarters of the patients (78\%) and there was no statistical difference in syrinx outcomes among any of the series subgroups [8]. This outcome suggests that the use of operative decompression techniques achieved appropriate treatment goals in the majority of the patients [5]. It should be noted, however, that about one-quarter of the patients (22\%) still did not experience improvement. Therefore, additional means to further improve syrinx outcomes in this patient subgroup should be investigated [8]. In a retrospective analyzed consecutive series of 66 patients operated by uniform posterior decompression, microsurgical reduction of the cerebellar tonsils, and dural closure using synthetic graft. 32 patients Chiari I malformation alone was present, and 34 in patients it was present in combination with syringomyelia. Clinical findings included pain, neurological deficits, and spinal deformity. The presence of syringomyelia was significantly associated with the presence of scoliosis. Radiological examination revealed normalization of tonsillar position in all patients. The syrinx had disappeared in 15 cases, was decreased in size in 17, and remained unchanged in two [9]. For our results, 15 (50\%) patients showed marked reduction in size, $3(10 \%)$ mild reduction in size 
and $12(40 \%)$ with no change.

Since Dr. Hans Chiari 1891 described Chiari type 1 malformation, several surgical procedures has been advocated for treatment of this anomaly, posterior fossa osseous decompression was basically needed either alone or in addition to many techniques as: durotomy, duroplasty, intradural dissection of arachnoid bands and outlet foramina of $4^{\text {th }}$ ventricle, plugging of the obex, tonsillar resection or shrinkage [10]. Syringomyelia associated with Chiari type 1 malformation was debated about optimum treatment procedure required as plugging of the obex \& placement of syringe-peritoneal shunt [11]. Cranio-cervical decompression alone became the preferred procedure since it is directed toward permanent elimination of the pathophysiology of this disorder with least risk [12]. In our study, posterior fossa decompression, durotomy, tonsillar shrinkage until restoration of its normal level, duroplasty with fascia lata was performed to all patients.

Regarding the extent of osseous resection, large posterior fossa craniectomy with extreme resection of the posterior edges of the foramen magnum laterally to the occipital condyles, followed by duroplasty without manipulation of the arachnoid was proposed, recording good results and minimal complication [13]. Others advocated much better results with small posterior fossa craniectomy which had a great importance preventing subsequent risk of cerebellar subsidence and recurrence of symptoms [10].

Durotomy without duroplasty was recommended with comparable results to other authors who insisted that duroplasty is essential to prevent postoperative scarring and maintain integrity of CSF flow around foramen magnum [14]. This was achieved in our study by grafting using fascia lata which is preferred than pericranum graft as it provides good area for dural grafting allowing enough room by its redundancy in the craniocervical area.

Intradural procedure, although not recommended by some authors who preferred leaving the arachnoid intact to prevent subarachnoid spillage of blood, arachnoiditis and re-adhesion [13]. Yet, these procedures are widely used by many authors who emphasized the importance of arachnoid opening, dissection of arachnoid bands around the area of foramen magnum, freeing CSF flow through outlet foramina of $4^{\text {th }}$ ventricle [15].

Sub-pial tonsillar resection was not attempted in our study and substituted with induction of tonsillar shrinkage by bipolar coagulation of the preserved pial covering of the tonsils, giving highly comparable results without risk of arterial injury e.g. PICA, although sub-pial tonsillar resection was reported to have good results in improving the volume mismatch of the posterior fossa [16].

Plugging of the obex was previously suggested relying on the hydrodynamic theory of Gardner for treating associated syringomyelia, but it was found to be ineffective treatment [17], in addition to its risk of brain stem injury, hence the procedure was excluded from our study reporting good results without any additional procedure in our scoop of follow up of the study. 
The treatment of type 1 Chiari malformation (CM-1) with posterior fossa decompression without (PFD) or with duroplasty (PFDD) is controversial. These options were analyzed (2000-2009) across the USA. PFDD patients were more likely White ( $81.2 \%$ vs $75.6 \%)$ and less likely admitted emergently (8.4\% vs. $13.8 \%)$. They also underwent more reoperations ( $2.1 \%$ vs. $0.7 \%)$, had more procedure-related complications ( $2.3 \%$ vs. $0.8 \%$ ), a longer length of stay (4.4 vs. 3.8 days) and higher charges (USD 35,321 vs. 31,483 ). This large study indicates that PFDD is performed more often in Caucasians, and associated with significantly more complications and immediate reoperations, while PFD is more frequent in those with syringomyelia and more economical, requiring fewer hospital resources. Overall, this study revealed that PFD is more favorable for CM-1, though it would be prudent to conduct a prospective trial [18].

In our study, the median complication rate was $6.2 \%$. The complications most commonly reported were CSF leak (2 cases) 6.66\%, pseudomeningocele (4 cases) $13.3 \%$, aseptic meningitis (2 cases) $6.66 \%$, wound infection (1 case) $3.33 \%$, meningitis ( 1 case) $3.33 \%$, neurological deficit (2 cases) 6.66\%, hydrocephalus and respiratory dysfunction ( 1 case) $3.33 \%$, postoperative headaches (5 cases) $16.7 \%$ and no epidural/subdural hematoma of the posterior cranial fossa. These are different from a study done on Pediatric and adult Chiari malformation Type I. The peak age of presentation in the adult series was 41 years, followed by 46 years. The incidence of syringomyelia was $65 \%$. In $92 \%$, the dura was opened, and in $65 \%$ of these cases, the arachnoid was opened and dissected. Tonsillar resection was performed in $27 \%$ of these patients. Postoperatively, syringomyelia improved or resolved in $78 \%$ of the patients. Postoperative neurological outcomes as follows: $75 \%$ improved, $17 \%$ showed no change, and $9 \%$ experienced worsening. Postoperative headaches improved or resolved in $81 \%$ of the patients, with a statistical difference in favor of the pediatric series. Postoperative complications were reported, most commonly with CSF leak, pseudomeningocele, aseptic meningitis, wound infection, meningitis, and neurological deficit, with a mean complication rate of $4.5 \%$. Complications were reported for $37 \%$ of pediatric, $20 \%$ of adult, and $43 \%$ of combined series. No difference in mortality rates was seen between the pediatric and adult series. The most common etiologies, in descending order of frequency, were pneumonia/respiratory failure, infection/sepsis, postoperative bleeding, and sleep apnea [5]. Also, the mortality of another study was very close to ours [3].

We have $1(3.33 \%)$ case of mortality among our cases, due to pneumonia/respiratory failure and infection/sepsis.

Finally, we recommend bony decompression of the posterior fossa with duroplasty using fascia lata after reduction of the size of the tonsils with watertight closure of the dura and patients and their families should be given clear information about the success of treatment and potential complications.

\section{Conflicts of Interest}

The authors declare no conflicts of interest regarding the publication of this paper. 


\section{References}

[1] Milhorat, T.H., Chou, M.W., Trinidad, E.M., et al. (2000) Chiari I Malformation Redefined: Clinical and Radiographic Findings for Symptomatic Patients. Neurosurgery, 44, 1005-1017. https://doi.org/10.1097/00006123-199905000-00042

[2] Iwasaki, Y., Hida, K., Koyanagi, I., et al. (2000) Reevaluation of Syringe-Subarachnoid Shunt for Syringomyelia with Chiari Malformation. Neurosurgery, 46, 407-413. https://doi.org/10.1097/00006123-200002000-00026

[3] Kamal, H.M. (2009) Posterior Fossa Decompression with Microscopic Fenestration of Foramen of Magendi and Duroplasty in Treatment of Adult Chiari Type 1 Malformation: Preliminary Results in 18 Consecutive Patients. Egyptian Journal of Neurosurgery, 24, 63-78.

[4] Aliaga, L., Hekman, K.E., Yassari, R., Straus, D., Luther, G., Chen, J., et al. (2012) A Novel Scoring System for Assessing Chiari Malformation Type I Treatment Outcomes. Neurosurgery, 70, 656-665. https://doi.org/10.1227/NEU.0b013e31823200a6

[5] Arnautovic, A., Splavski, B., Boop, F.A., Arnautovic, K.I., et al. (2015) Pediatric and Adult Chiari Malformation Type I Surgical Series 1965-2013: A Review of Demographics, Operative Treatment, and Outcomes. Journal of Neurosurgery. Pediatrics, 15, 161-177. https://doi.org/10.3171/2014.10.PEDS14295

[6] Hayhurst, C., Richards, O., Zaki, H., Findlay, G. and Pigott, T.J. (2008) Hindbrain Decompression for Chiari-Syringomyelia Complex: An Outcome Analysis Comparing Surgical Techniques. British Journal of Neurosurgery, 22, 86-91. https://doi.org/10.1080/02688690701779525

[7] Haroun, R.I., Guarnieri, M., Meadow, J.J., Kraut, M. and Carson, B.S. (2000) Current Opinions for the Treatment of Syringomyelia and Chiari Malformations: Survey of the Pediatric Section of the American Association of Neurological Surgeons. Pediatric Neurosurgery, 33, 311-317. https://doi.org/10.1159/000055977

[8] Zhang, Z.Q., Chen, Y.Q., Chen, Y.A., Wu, X., Wang, Y.B. and Li, X.G. (2008) Chiari I Malformation Associated with Syringomyelia: A Retrospective Study of 316 Surgically Treated Patients. Spinal Cord, 46, 358-363. https://doi.org/10.1038/sj.sc.3102141

[9] Alzate, J.C., Kothbauer, K.F., Jallo, G.I. and Epstein, F.J. (2001) Treatment of Chiari I Malformation in Patients with and without Syringomyelia: A Consecutive Series of 66 Cases. Neurosurgical Focus, 11, E3.

[10] Klekamp, J., Batzdorf, U., Samii, M. and Bothe, H.W. (1996) The Surgical Treatment of Chiari I Malformation. Acta Neurochirurgica (Wien), 138, 788-801. https://doi.org/10.1007/BF01411256

[11] Iwasaki, Y., Hida, K., Koyanagi, I., et al. (2000) Reevaluation of Syringosubarachnoid Shunt for Syringomyelia with Chiari Malformation. Neurosurgery, 46, 407-413. https://doi.org/10.1097/00006123-200002000-00026

[12] Oldfield, E.H. (2002) Cerebellar Tonsils and Syringomyelia. Journal of Neurosurgery, 97, 1009-1010. https://doi.org/10.3171/jns.2002.97.5.1009

[13] Sindou, M., Chavez-Machuca, J. and Hashish, H. (2002) Cranio-Cervical Decompression for Chiari Type I Malformation Adding Extreme Lateral Foramen Magnum Opening and Expansile Duraplasty with Arachnoid Preservation. Technique and Long-Term Functional Results in 44 Consecutive Adult Cases Comparison with Literature Data. Acta Neurochirurgica (Wien), 144, 1005-1019. https://doi.org/10.1007/s701-002-8289-9

[14] Krieger, M.D., Mccomb, J.G. and Levy, M.L. (1999) Toward a Simpler Surgical 
Management of Chiari I Malformation in a Pediatric Population. Pediatric Neurosurgery, 30, 113-121. https://doi.org/10.1159/000028777

[15] Munshi, I., Firm, D., Stine-Reyes, R., et al. (2000) Effects of Posterior Fossa Decompression with \& without Duroplasty on Chiari Malformation Associated Hydromyelia. Neurosurgery, 46, 1384-1390. https://doi.org/10.1097/00006123-200006000-00018

[16] Guyotat, J., Bret, P., Jouanneau, E., et al. (1998) Syringomyelia Associated with Type I Malformation. A 21-Year Retrospective Study on 75 Cases Treated by Foramen Magnum Decompression with a Special Emphasis on the Value of Tonsils Resection. Acta Neurochirurgica, 140, 745-754. https://doi.org/10.1007/s007010050175

[17] Gardner, W.J. and Angel, J. (1959) The Mechanism of Syringomyelia \& Its Surgical Correction. Clinical Neurosurgery, 6, 131-140.

https://doi.org/10.1093/neurosurgery/6.CN suppl 1.131

[18] Shweikeh, F., Sunjaya, D., Nuno, M., Drazin, D. and Adamo, M.A. (2014) National Trends, Complications, and Hospital Charges in Pediatric Patients with Chiari Malformation Type I Treated with Posterior Fossa Decompression with and without Duraplasty. Pediatric Neurosurgery, 50, 31-37.

https://doi.org/10.1159/000371659 